\title{
Democratic Policy-making for Misinformation Detection Platforms by Git-based Principles ${ }^{1}$
}

\author{
Oul Han*, Ipek Baris*, Akram Sadat Hosseini", \\ Sarah de Nigris ${ }^{*}$ and Steffen Staab ${ }^{*}$, \\ "Institute for Web Science and Technologies (WeST), \\ University of Koblenz, Germany han@uni-koblenz.de, \\ ibaris@uni-koblenz.de, sadathosseini@uni-koblenz.de, \\ denigris@uni-koblenz.de, staab@uni-koblenz.de \\ ${ }^{\dagger}$ Web and Internet Science Group (WAIS), University of Southampton, \\ United Kingdom
}

\begin{abstract}
Combating misinformation is a challenging task due to the fact that misinformation evolves in content and strategy. We describe the challenges of this task and propose a git-based framework for collaborative and open policymaking against ever-evolving misinformation. We present the setup for future test-runs where users receive tasks that conduct the core functions of git-based policy-making against misinformation.
\end{abstract}

\section{Keywords}

Co-creation $\cdot$ Git $\cdot$ policy development $\cdot$ open governance $\cdot$ misinformation

${ }^{1}$ Supported by EU-Project Co-Inform under grant agreement No 770302.

How to cite this book chapter:

Han, O., Baris, I., Hosseini, A.S., de Nigris, S. and Staab, S. 2020. Democratic

Policy-making for Misinformation Detection Platforms by Git-based Principles. In: Loizides, F., Winckler, M., Chatterjee, U., Abdelnour-Nocera, J. and Parmaxi, A. (eds.) Human Computer Interaction and Emerging Technologies: Adjunct Proceedings from the INTERACT 2019 Workshops. Pp. 67-74. Cardiff: Cardiff University Press. DOI: https://doi.org/10.18573/book3.h. License: CC-BY 4.0. 


\section{Introduction}

Misinformation in online media is a broad term to design deceitful content, such as disinformation (i.e. fake news), rumors, manipulated content, authentic material used in the wrong context [6]. While misinformation in general may not be driven all the time by the intent to deceit, disinformation has indeed such aim [2].

Under any form, however, misinformation undeniably poses a threat, as this content can maliciously manipulate peoples beliefs and their decisions, carrying thus a social impact. For example, misinformation about the refugee crisis affected how citizens view refugees and their attitudes towards national and European Union politics [1]. Countering the instrumental use of misinformation to manipulate the public opinion is a multifaceted challenge: policy design to this end spreads on many levels, starting from the very definition and detection of misinformation to the regulation of online platform users' behaviour. Thus, our contribution revolves around the following research question: How to improve platform-internal management policies against misinformation that is spread by users of diverse backgrounds, which negatively affects all platform users?

Online platforms, such as Twitter and Facebook, provide, indeed, the natural environment for the aforementioned challenge and many, if not all, of them have already undertaken such task of policy design after having been a fertile substrate for misinformation diffusion. For example, Facebook has a section called "community standards" which lay out common policies. ${ }^{2}$ It includes a dedicated paragraph on how they will combat the spread of false news on their platform, and lists a number of methods by which they seek better regulation. Stated among these methods are the disruption of economic incentives for spreading misinformation, using machine learning for false news detection, and integrating third-party fact-checkers. Other comparable platforms list their policies for the management of platform users and misinformation in similar format, which is the result of centralized policy-making by a closed minority of platform managers, developers, and governments.

However, this top-down approach is the dangerous Achilles' heel of such policies: We argue that, to target and effectively manage the diverse types of misinformation via democratic participation (i.e. "acts that are intended to influence the behavior of those empowered to make decisions" [5, p. 53]), the policy design process should be a decentralized and collaborative one, to allow the open inclusion of platform users, instead of being opaquely determined by a small group of experts in public or private sectors.

Thus, in this position paper, we propose a git-based framework to enable such collaborative and flexible policy-making, which we describe in Sec. 3.1. Moreover, we propose is Sec 3.3 how this framework could be tested by users, who will be given initial policies and specific tasks that relate to the further development of policies according to their wants and needs.

${ }^{2}$ https://www.facebook.com/communitystandards/false_news/. 


\section{Related Work}

Governments and public institutions are using Git-based frameworks for open co-creation of computer code as well as codified text (laws and policies). Audrey Tang, the current Digital Minister of Taiwan and civic hacker, provides git repositories for open government tools with the call to "fork the government". The NYU GovLab's Project CrowdLaw ${ }^{4}$ seeks to involve collective intelligence in every stage of lawmaking, and mentions more than two dozen examples worldwide where governments use the Internet to involve citizens for proposing legislation, drafting bills, monitoring implementation, and supplying missing data.

Some even go further and ask: "What if anyone could write amendments to existing laws, or even entirely new laws and propose them to Congress (or lobby their Congressperson to introduce it) using pull requests?" 5 This idea is already nearly fully implemented: San Francisco laws, ${ }^{6}$ the White House Open Data policy, ${ }^{7}$ and government agency services ${ }^{8}$ are forkable. These and more repositories by official government institutions around the world are listed in the Government GitHub Community. ${ }^{9}$ 10k active government users were reported in 2014 with steep trend ${ }^{10}$. Studies provide user and satisfaction surveys with usage statistics that imply that git-based co-creation of textual policy (as opposed to software code) is useful for general collaboration [3-4].

In addition but also in contrast, we highlight a specific application area for git-based co-creation of textual policy against multi-medial and multi-lingual misinformation on online platforms. We argue that git-based co-creation of textual policies makes immediate sense for the case of platform policy for antimisinformation, because online platforms merge the application area (the Internet and its information environment) with the target of policies (online misinformation), which differs from the online co-creation of offline laws. Additionally, the national and contextual diversity of misinformation is its largest challenge and is well addressed by branching out policies across authors from various backgrounds.

In open co-creation, most likely complications are: The higher the openness, the freedom to co-create is higher, and the risk of disagreement between users

${ }^{3} \mathrm{http}: / / \mathrm{g} 0 \mathrm{v} \cdot \mathrm{asia} /$.

${ }^{4} \mathrm{http}: / /$ www.thegovlab.org/project-crowdlaw.html.

${ }^{5}$ https://blog.abevoelker.com/gitlaw-github-for-laws-and-legal-documents-a -tourniquet-for-american-liberty/.

${ }^{6}$ https://github.com/SFMOCI/openlaw.

${ }^{7}$ https://project-open-data.cio.gov/.

${ }^{8}$ https://github.com/cfpb/transit_subsidy/pull/1\#commits-pushed-323f076.

${ }^{9} \mathrm{http}: / /$ government.github.com/community/.

${ }^{10} \mathrm{https} / /$ github.blog/2014-08-14-government-opens-up-10k-active -government-users-on-github/. 
is higher regarding a) which misinformation to regulate how and b) how to regulate co-creating users in the case where subjective views collide.

\section{Organizing policy-making against misinformation}

Our approach proposes a decentralized and horizontal git-based framework for misinformation policy-making in an online platform. We describe functions, examples, and the testing setup.

\subsection{Git-based functions}

If the creation of policies does not involve platform users, policies will lag behind real misinformation, or miss blind spots that are outside the range of expertise, cultural familiarity, or linguistic barriers of a closed, centralized minority of policy-makers. Moreover, the centralization of such policy-design by a minority can pose a potential threat to the freedom of speech, as it would be in charge of discerning what is misinformation and what is not.

In this view, the native functions of git ${ }^{11}$ allow to bypass such limitations, implementing decentralized and democratic policy-making through the following actions:

- Version control enables tracking changes of the project/written code, which can be a set of written misinformation management policies.

- Push is used for updating a project, and pull is used for accepting changes in a project. These functions enable developers to work collaboratively while users can develop policies with complete freedom, while remaining connected to the updated version of the original root, with the option to communicate or merge at any time.

- Clone copies an existing project into a freely modifiable copy of the project.

This gives flexibility to developers for working on the project in their own server. This functionality can be applied for an open and decentralized development of misinformation management policies. If clone is utilized by users from different backgrounds, the handling of misinformation can differ by culture and country's specific regulations (i.e it is likely that offensive content will be different and/or unexpected within cultures, or nations).

\subsection{Example Usecase}

An initial and generic example policy could be: "We inspect posts that contain hate speech against minorities". A user finds that some posts are not against

${ }^{11}$ https://git-scm.com/docs/. 
minorities but are satirical (e.g. in liberal left-leaning satire), and decides that the policy needs conditions. Hence, she suggests the following revision: "We inspect posts that contain hate speech against minorities, if the hate speech is not irony". Another user still finds flaws in this formulation, because in his resident country, this sort of satire does not exist. Now, he and any other users may either suggest revisions, or develop their own version that fits their own information environments, national and cultural conflicts, or linguistic traits. ${ }^{12}$

The above example policy-making process starts from initial policy, then is followed by specification by user A, which is followed by further specification by user B. Additionally, user B forks the policy for further modifications that diverge from the original specifications. This entire process is facilitated by git-based principles and Github-based social interactions for deciding platform policies that manage both misinformation content and user regulation (e.g Figure 1.)

\subsection{Setup of the testing environment}

We provide the environment for test users by setting up a Git-based social media platform. For trials, we provide initial platform policies. Each policy has its own folder for separate development. In this framework, policies are not just written but developed. The participants of our trials are stakeholders of different backgrounds (e.g occupation, age, culture). We ask the participants to perform following actions:

- Create national versions of policies

- Creating/editing policies on main repository

- Discussion for better misinformation coverage by policies

- Clone main repository and make revision for desired policy

- Offer revised suggestions by push/pull requests

- Accept revised suggestions by moderator pull

- Discuss the role of moderator, then create a policy for moderators

A git-based framework presents the codification of policies as a collaborative coding project. In order to increase the accessibility for users to the git based framework, we rename git-specific-functions with terms that describe their policy-specific function. Table 1 shows our suggestions and maps each function to democratic effects with positive and negative implications. ${ }^{13}$ Additional GitHubnative functions are shown that relate to graphical interfaces and user interactions.

12 All policies are automated by the platform, which performs machine readable rules, such as: "In the event of a post containing a word from this list of hate speech, alert this user".

${ }^{13}$ https://www.opengovpartnership.org/glossary. 


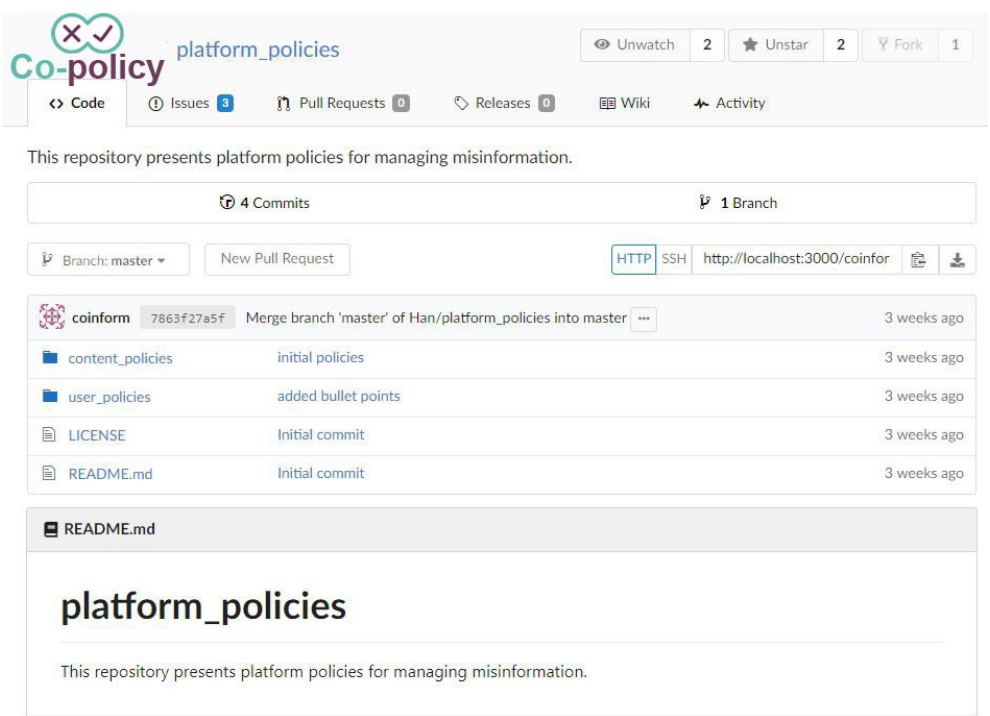

\section{\#2 Policy for moderator needed \\ (1) Open opened 3 weeks ago by Han 1 comments}

Issues like these will need a moderator. The moderator will have additional roles such as resolving issues or deciding as last instance. What is the co-creation way (read: democratic way) of deciding a moderator and determining the moderator's "term"? Will it be a rotating system, or similar to direct voting?

Lis 1

I agree with you about moderator. Do you think full-automated moderator could work?

Write Preview

Fig. 1: Git-based platform where users edit and discuss misinformation policies.

Finally, we will conduct data analytics on the resulting policies and satisfaction survey on user experiences. Test runs should yield two levels of policy: misinformation content and user management.

1. Misinformation content

- Which contents of misinformation and which handling actions were covered by the users?

- Was the process of developing policies more interactive in cases of agreement or disagreement? 
Table 1: Mapping the name of git-based (and GitHub-only) functions to policymaking

\begin{tabular}{|l|l|l|}
\hline Git & \multicolumn{1}{|c|}{$\begin{array}{c}\text { Policy-making } \\
\text { function }\end{array}$} & \multicolumn{1}{c|}{$\begin{array}{c}\text { Democratic effect (positive/ } \\
\text { negative implication) }\end{array}$} \\
\hline $\begin{array}{l}\text { software code } \\
\text { moderating } \\
\text { version control }\end{array}$ & $\begin{array}{l}\text { policy } \\
\text { moderate } \\
\text { track-policy }\end{array}$ & $\begin{array}{l}\text { transparency (information/ } \\
\text { too much information) }\end{array}$ \\
\hline $\begin{array}{l}\text { blame } \\
\text { commit merge }\end{array}$ & $\begin{array}{l}\text { whose-idea } \\
\text { contribute finalize }\end{array}$ & $\begin{array}{l}\text { accountability } \\
\text { (shared control/need for control) }\end{array}$ \\
\hline $\begin{array}{l}\text { clone } \\
\text { push pull } \\
\text { diff branch }\end{array}$ & $\begin{array}{l}\text { take update } \\
\text { accept difference } \\
\text { localize-policy }\end{array}$ & $\begin{array}{l}\text { public engagement } \\
\text { (inclusiveness/conflicting perspectives) }\end{array}$ \\
\hline $\begin{array}{l}\text { GitHub-only } \\
\text { discussion } \\
\text { issue report }\end{array}$ & $\begin{array}{l}\text { discussion } \\
\text { suggest-policy }\end{array}$ & $\begin{array}{l}\text { public engagement (discussion/failure } \\
\text { of agree) }\end{array}$ \\
\hline
\end{tabular}

- How were discussions resolved at disagreement over defining and handling misinformation?

2. User management

- What is the best policy for electing and managing moderators?

- What is the best policy for managing conflicting views in the process of defining policies?

\section{Conclusion}

This position paper proposes git-based framework for developing platform policies on misinformation in a decentralized and collaborative way. We introduce the benefits of git for misinformation policy-making for platforms, and suggest a methodology for testing the requirements. As future work, we will evaluate the outcomes by feedback rounds and conduct data analysis on users interactions.

\section{References}

1. Bartsch, M., Clau, A.: The Case of the Murdered Goats: Exploring Germany's Far-Right Rumor Mill. Spiegel Online (Jan 2016), https://www.spiegel.de /international/germany/far-right-misinformationstokes-anti-refugee -sentiment-a-1070413.html

2. Golbeck, J., Mauriello, M., Auxier, B., Bhanushali, K.H., Bonk, C., Bouzaghrane, M.A., Buntain, C., Chanduka, R., Cheakalos, P., Everett, J.B., 
et al.: Fake news vs satire: A dataset and analysis. In: Proceedings of the 10th ACM Conference on Web Science. pp. 17-21. ACM (2018)

3. Longo, J., Kelley, T.M.: Github use in public administration in canada: Early experience with a new collaboration tool. Canadian Public Administration 59(4), 598-623 (2016)

4. Mergel, I.: Open collaboration in the public sector: The case of social coding on github. Government Information Quarterly 32(4), 464-472 (2015)

5. Verba, S.: Democratic participation. The Annals of the American Academy of Political and Social Science 373(1), 53-78 (1967)

6. Wardle, C.: 6 types of misinformation circulated this election season. Columbia Journalism Review 18 (2016) 